\title{
Structural and Chemical Properties of ZnSe-Fe Electrodeposited Granular Films
}

\author{
A.R. de Moraes, D.H. Mosca, W.H. Schreiner, N. Mattoso, and E. Silveira \\ Departamento de Física - UFPR, Centro Politécnico, CP 19044 \\ 81531-990 Curitiba-PR, Brazil
}

Received on 23 April, 2001

\begin{abstract}
Potentiostatic electrodeposition methods have been used to grow polycrystalline ZnSe and ZnSe-Fe granular thin films on stainless steel substrates from aqueous solutions. These films were characterized by x-ray diffraction, transmission electron microscopy, x-ray photoelectron spectroscopy and Raman spectroscopy.
\end{abstract}

\section{Introduction}

Hybrid semiconductor (SC)/ ferromagnetic (FM) structures have been investigated due to the possibility of using not only the charge but also the spin of electrons to conceive new electronic devices [1-3]. In particular, ZnSe has been pointed out as one attracting candidate to achieve such goal due to their favorable chemical stability in the presence of Fe [4] and theoretical calculations suggesting that large tunnel magnetoresistance could be attained [5]. Currently, most of hybrid SC/FM systems are fabricated using molecular beam epitaxy (MBE) [6,7]. However, the simpler and inexpensive electrodeposition of II-VI semiconductor compounds such as ZnSe has already been studied for applications in optoelectronics and solar cells [8-11]. In this work we report for the first time on the fabrication of the $\mathrm{ZnSe}-\mathrm{Fe}$ granular thin films by electrodeposition techniques.

\section{Sample preparation}

ZnSe-Fe granular thin films were electrodeposited under potentiostatic conditions onto mechanically and chemically polished stainless steel substrates. All deposition experiments were performed using a EG\&G PAR $273 \mathrm{~A}$ galvanostat/potentiostat with a stationary parallel plate electrode system consisting of a platinum disk counter-electrode and a saturated $\mathrm{Ag} / \mathrm{AgCl}$ reference electrode. Plating solutions were prepared with reagent grade chemicals $\left(\mathrm{ZnSO}_{4}, \mathrm{SeO}_{2}\right.$, and $\left.\mathrm{Fe}\left(\mathrm{NH}_{4}\right)_{2}\left(\mathrm{SO}_{4}\right)_{2}\right)$ immediately prior to each experiments by dissolving the requisite amount of the metallic components in distilled water. All samples were deposited at $65{ }^{\circ} \mathrm{C}$ under a ca- thodic potentials with $\mathrm{pH}$ value adjusted between 2.0 and 2.5 by using sulfuric acid as additive. Pure $\mathrm{ZnSe}$ thin films were deposited at $-0.85 \mathrm{~V}$ from solutions containing $200 \mathrm{mM} \mathrm{Zn}$ and $1 \mathrm{mM}$ Se. As reported in the literature [8-11], the ZnSe thin films obtained in these conditions are metallic gray, crystallized with a dense morphology. Incorporation of Fe in the solution (partial concentration between 0.1 to $10 \mathrm{mM}$ ) does not significantly change, such physical and morphological, the deposits according to optical and scanning electron microscopy analyses. All the films are micrometer thick. A detailed investigation of the simultaneous electrodeposition of the $\mathrm{Zn}$, Se and Fe ions will be presented elsewhere [12].

\section{Results and Discussion}

X-ray Photoemission Spectroscopy (XPS) analyses show that the ZnSe thin films are free of contaminants within chemical sensibility (about 1 at. \%) of our experiments performed in an ESCA3000 VG system. From XPS profile analyses performed by $\mathrm{Ar}^{+}$ion bombardment at $3 \mathrm{keV}$, an excess of Se is observed near the surface of the deposits. Since the $\mathrm{Ar}^{+}$ion bombardment of the $\mathrm{ZnSe}$ is congruent [13]; i.e., the stoiquiometry of the ZnSe compound is maintained, we conclude that the excess of Se is related to the electrochemical kinetics. XPS analyses reveals that ZnSe films prepared from solutions containing low Fe concentration exhibits similar Se excess at the surface. After surface etching with $\mathrm{Ar}^{+}$ions, an oxygen-free surface is obtained and the Zn2p and Se2p core-level signals tend to ZnSe stoichiometry. Fig. 1 shows a characteristic x-ray diffraction pattern of a sample with $100 \mathrm{mM}$ Fe in the plating 
solution. Together with the intense diffraction peaks of the substrate, three diffraction peaks observed at $31.7^{0}$, $53.2^{0}$, and $63^{\circ}$ are associated with cubic ZnSe with a zincblende structure [14]. The diffraction peaks around $27.2^{0}$ and $66.4^{0}$ were identified as Se with an hexagonal structure whereas another peak around $34.6^{0}$ reveals the presence of the $\mathrm{SeO}_{2}$ [14]. The trigonal Se formation cannot be ruled out [15]. Thus, the sample is polycrystalline with a predominant ZnSe (111)texture along the growth direction. The absence of the Fe diffraction peaks are probably due to small particle size and their intrinsic structural disorder since some $\mathrm{Fe}$ diffraction rings are visible in the Transmission Electron Microscopy (TEM) analyses. TEM analyses were performed using a JEOL JEM 1200II electron microscope operating at $120 \mathrm{kV}$. For TEM analyses the stainless steel substrate were removed from the backside of the samples by controlled electrochemical etching using a diluted nitric acid solution. In Fig. 2(a) is shown a bright field image of a pure ZnSe deposit. Selected Area electron Diffraction (SAED) pattern of this same region is shown in Fig. 2(b). Some electron diffraction rings of the $\mathrm{ZnSe}$ are identified. Fig. 2(c) shows a bright field image of a deposit prepared with $1 \mathrm{mM}$ Fe. According to the electronic density contrast, the Fe-rich regions (dark zones) are dispersed in a $\mathrm{ZnSe}$ template. Fe-rich particles having average diameters varying from $20 \mathrm{~nm}$ to $100 \mathrm{~nm}$ are observed. The network of the Fe-rich particles exhibits a broad size distribution. The same electrodeposit exhibit percolating regions (not shown) with Fe-rich clusters having an average diameter size as large as $950 \mathrm{~nm}$. Fig. 2(d) showns the SAED pattern of the same region of the sample shown in the Fig. 2(c). Some diffraction rings associated with $\mathrm{ZnSe}$ and Fe are identified. Therefore, a network of Fe-rich granules almost physically uncoupled between them can be obtained by electrochemical process from aqueous solutions with concentration smaller than $1 \mathrm{mM}$ Fe. Using a Jobin-Yvon T64000 micro-Raman system, the Raman scattering measurements were performed at room temperature in the backscattering configuration from the sample growth surface. After dispersed by the spectrometer the scattered light was detected by a liquid nitrogen cooled charge coupled device. The Raman spectra were excited by the $514.5 \mathrm{~nm}$ line of a $\mathrm{Ar}^{+}$ laser. The laser was focused to a spot size of about 2 $\mu \mathrm{m}$ and the power density was kept under $104 \mathrm{~W} / \mathrm{cm}^{2}$, in order to avoid damage to the samples. No polarization analyzer was used in order to select the scattered light coming from the sample. The spectral resolution was $4 \mathrm{~cm}^{-1}$. Fig. 3 depicts the Raman spectra of four samples grown under different conditions specified in its caption. The spectra were shifted vertically for the

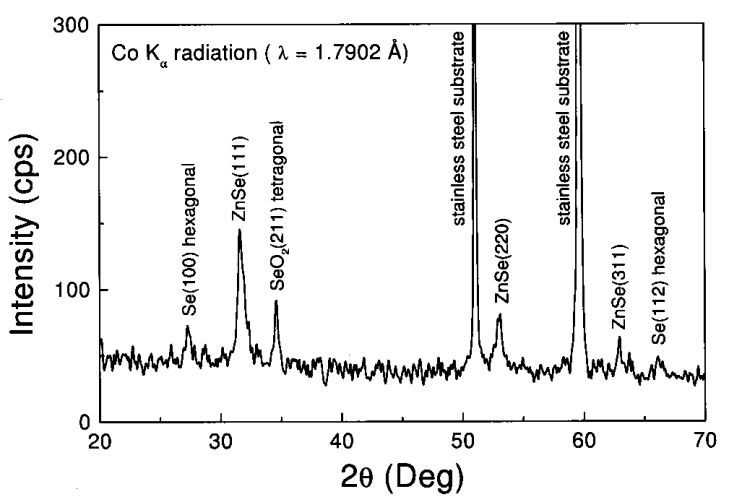

Figure 1. X-ray diffraction pattern in the Bragg-Brentano geometry performed with $\mathrm{Co} \mathrm{K}_{\alpha}$ radiation for a $\mathrm{ZnSe}-\mathrm{Fe}$ electrodeposit with $100 \mathrm{mM}$ Fe in the solution.

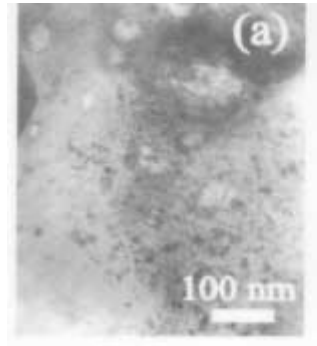

$\mathrm{ZnSe}$

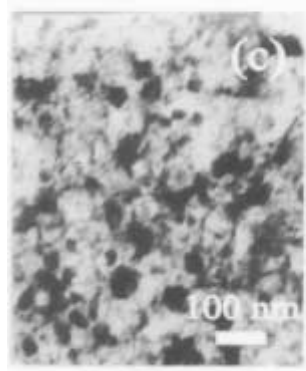

$\mathrm{ZnSe-Fe}$

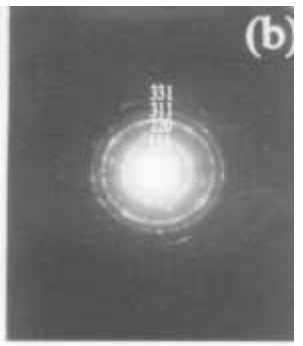

ZnSe

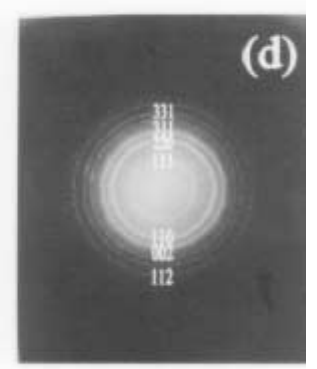

$\mathrm{ZnSe-Fe}$
Figure 2. (a) and (c) Bright field micrographs of pure ZnSe and $\mathrm{ZnSe}-\mathrm{Fe}$ prepared from solutions containing $1 \mathrm{mM} \mathrm{Fe}$, respectively. (b) and (d) Selected-area electron diffraction pattern of the same regions reported in (a) and (c), respectively. Some diffraction rings are identified.

sake of clarity. We expect an increasing of Fe incorporation in the samples from (a) to (d), with pure $\mathrm{ZnSe}$ being labeled as (a). The spectrum of pure $\mathrm{ZnSe}$ consists of a longitudinal optical (LO) phonon at $252 \mathrm{~cm}^{-1}$ and the transverse mode (TO) at about $205 \mathrm{~cm}^{-1}$ [16]. For all the spectra showed in Fig. 3 we observe one structure at $252 \mathrm{~cm}^{-1}$ and a weak leakage from the TO at $205 \mathrm{~cm}^{-1}$. The pronounced peak at about $235 \mathrm{~cm}^{-1}$ can be attributed to a trigonal Se phase [17], since an excess of Se on top of a stoichiometric ZnSe template was observed by XPS and x-ray diffraction analyses. The Raman spectrum taken from the substrate showed no modes in this region. ZnSe TO-LO peak positions remains unchanged for all the samples, therefore no 
formation of intermediary compounds is expected [16]. The second order zone-boundary $2 \mathrm{TA}(\mathrm{X})$ mode can be observed at about $140 \mathrm{~cm}^{-1}$. With the increase of $\mathrm{Fe}$ content the range under $150 \mathrm{~cm}^{-1}$ becomes more pronounced indicating an increase of disorder in the samples. With increase of Fe content one observes also an increase in the scattered intensity for the $\mathrm{ZnSe} \mathrm{LO}$ and the TO phonons, in comparison with the mode at 235 $\mathrm{cm}^{-1}$. As the Raman measurements, SAED pattern and X-ray diffraction analyses showed no evidence of intermediary compounds of the type $\mathrm{Zn}_{1-x} \mathrm{Fe}_{x}$ Se, we do not expect change in the gap of the material and consequently resonance effects. We attribute this effect as coming from surface enhanced Raman scattering due to the formation of Fe granules inside the sample [18]. In conclusion, we demonstrate that is possible the fabrication of granular $\mathrm{ZnSe}-\mathrm{Fe}$ thin films with a polycrystalline structure using electrochemical processes.

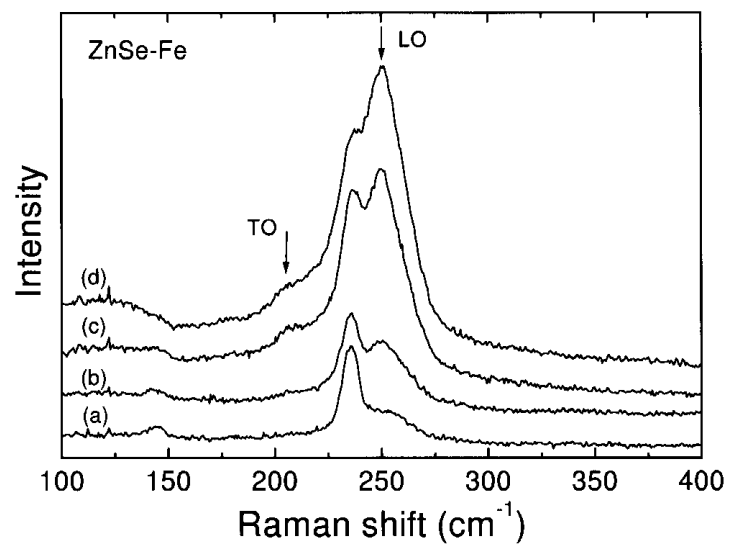

Figure 3. Raman spectra of (a) pure ZnSe deposited at -0.85 $\mathrm{V} ; \mathrm{ZnSe}-\mathrm{Fe}$ deposited under periodically pulsed potentials between $-0.85 \mathrm{~V}$ and $-1.1 \mathrm{~V}$ for times (b) $1 \mathrm{~s}$ and $0.1 \mathrm{~s}$ from solution containing $1 \mathrm{mM} \mathrm{Fe}$; (c) $1 \mathrm{~s}$ and $0.1 \mathrm{~s}$ from solution containing $100 \mathrm{mM} \mathrm{Fe}$ and (d) 1s and 1s from solution containing $100 \mathrm{mM} \mathrm{Fe}$.

\section{Acknowledgments}

The authors are grateful to Centro de Microscopia Eletrônica (UFPR) for the technical support in the TEM measurements and to Group of Optical Properties (IFGW-UNICAMP) for their help with the Raman measurements. This work was partially supported by CNPq and PRONEX/MCT.

\section{References}

[1] R. Fiederling, M. Keim, G. Reuscher, W. Ossau, G. Schmidt, A. Waag, L. W. Molenkamp, Nature 402, 787 (1999).

[2] H. Ohno, Science 281, 951 (1998).

[3] G. A. Prinz, Phys. Today 48(4), 58 (1995).

[4] P. Walser, M. Hunziker, T. Speck, M. Landolt, Phys. Rev. B 60, 4082 (1999).

[5] J. M. MacLaren, X. G. Zhang, W. H. Butler, X. Wang, Phys. Rev. B 59, 5470 (1999).

[6] D. H. Mosca, J. M. George, J. L. Maurice, A. Fert, M. Eddrief, V. H. Etgens, J. Magn. Magn. Mater. 226, 917 (2001).

[7] E. Reiger, E. Reinwald, G. Garreau, M. Ernst, M. Zölfl, E. Bensch, S. Bauer, H. Preis, G. Bayreuther, J. Appl. Phys. 87, 5923 (2000).

[8] A. P. Samantileke, M. H. Boyle, J. Young, I. M. Dharmadasa, J. Mater. Sci.: Mater. Electron. 9, 231 (1998).

[9] R. Chandramohan, C. Sanjeeviraja, T. Mahalingam, Phys. Stat. Sol. (a) 163, R11.C (1997).

[10] C. Natarajan, M. Sharon, C. Lévy-clément, M. Neumann-Spallart, Thin Solid Films 237, 118 (1994).

[11] V. Khrishnan, D. Ham, K. K. Mishra, and K. Rajeswar, J. Electrochem. Soc. 139, 23 (1992).

[12] A. R. de Moraes, D. H. Mosca, N. Mattoso, W. H. Schreiner, Electrochem. Solid-St. Lett. 5, xxxx (2002).

[13] I. M. Dharmadasa, C. J. Blomfield, G. E. Gregory, B. C. Cavenett, K. A. Prior, J. Simpson, Surf. Interf. Anal. 21, 718 (1994).

[14] File PDF 37-1463, ICDD International Centre for Diffraction Data, Newtown Square, PA, 1999.

[15] R. M. Marin, G. Lucovsky, K. Hellwell, Phys. Rev. B 13, 1383 (1976).

[16] C.-L. Mak, R. Sooryakumar, B. T. Jonker, G. A. Prinz, Phys. Rev. B 45, 3344 (1992).

[17] V. V. Poborchii, A. V. Kolobov, K. Tanaka, Appl. Phys. Lett. 72, 1167 (1998).

[18] P. G. Cao, J. L. Yao, B. Ren, B. W. Mao, R. A. Gu, Z. Q. Tian, Chem. Phys. Lett. 316, 1 (2000). 